\title{
Prospective investigation of pain associated with ultrasound- and computed tomography-guided percutaneous biopsies in oncological patients
}

\author{
Andrei Roman ${ }^{1}$, Federica Palego ${ }^{1}$, Patriciu Achimas-Cadariu ${ }^{2}$, Catalin Vlad ${ }^{2}$, Alexandra \\ Andries ${ }^{1}$, Ciprian Ionut Tomuleasa ${ }^{3}$, Vlad Alexandru Gata ${ }^{2}$, Roxana Pintican ${ }^{4}$, Maria \\ Patricia Rada ${ }^{5}$, Diana Feier ${ }^{4}$
}

${ }^{1}$ Radiology Department, "Prof. Dr. Ion Chiricuță" Oncology Institute, ${ }^{2}$ Surgery Department, "Prof. Dr. Ion Chiricuţă" Oncology Institute, ${ }^{3}$ Hematology Department, "Prof. Dr. Ion Chiricuţă" Oncology Institute, ${ }^{4}$ Radiology Department, County Emergency Hospital Cluj-Napoca, ${ }^{5}$ Second Gynecology Department, County Emergency Hospital ClujNapoca, "Iuliu Hatieganu" University of Medicine and Pharmacy, Cluj-Napoca, Romania

\begin{abstract}
Aims: We aimed to investigate the pain, anxiety and other factors that are associated with percutaneous computed tomography (CT)- and ultrasound (US)-guided biopsies of tumors. Material and methods: The study prospectively included 60 consecutive patients, of which 24 underwent CT-guided biopsies and 36 US-guided biopsies. The location of targeted tumors was within the thorax, abdomen, pelvis, and limbs. A questionnaire containing numerical rate scales $(0-10)$ regarding procedural and post-procedural pain, anxiety and other associated parameters was filled out by each patient 2-6 hours after the procedure. CT and US parameters were compared. The two groups were then pooled together in order to compare pain scores per targeted organ and to analyze the parameters that were associated with pain. Results: There was no significant difference between the CT and US group with the exception of the positional discomfort, which was higher in the CT group ( $\mathrm{p}=0.003$ ). The average procedural pain score (2.0) was significantly higher than the post-procedural pain $(1.3, \mathrm{p}=0.006)$ and the phlebotomy pain $(0.8, \mathrm{p}<0.0001)$. There was no significant difference between the targeted organs with regards to the pain score. The factors that showed a positive correlation with the procedural pain were procedure-related anxiety $(\mathrm{p}=0.005)$, positional discomfort $(\mathrm{p}=0.01)$ and phlebotomy pain $(\mathrm{p}=0.0008)$. The pre interventional use of an analgesic was negatively correlated with the procedural pain $(\mathrm{p}=0.02)$. Conclusions: CT- and US- guided percutaneous biopsies are associated with low levels of pain that are generally well tolerated by patients irrespective of the targeted organ.
\end{abstract}

Keywords: ultrasonography; CT; biopsy; tumor; pain

\section{Introduction}

Tumor biopsy is one of the cornerstones of current oncological practice. This procedure is essential not only

Received 09.11.2019 Accepted 19.01.2020

Med Ultrason

2020, Vol. 22, No 1, 65-70

Corresponding author: Andrei Roman, MD, PhD

Radiology Department, "Prof. Dr. Ion Chiricuţă"

Oncology Institute, "Iuliu Hatieganu" University

of Medicine and Pharmacy, Cluj-Napoca,

Romania, 39 Buna Ziua street,

400495 Cluj-Napoca, Romania

Phone: 0740021454

E-mail: Andrei.roman678@gmail.com during initial diagnosis, but it is also being increasingly required over the course of the disease for optimal treatment guidance. Percutaneous biopsies are usually performed under ultrasound (US) or computed tomography (CT) guidance in any part of the body, except the central nervous system and the tubular organs that are more easily accessible by endoscopy. The guidance method choice depends on the size, location and visibility of the tumor, but also on the operator's preference, US being usually selected due to the lack of radiation. However, some lesions may be accessible only with the aid of CT guidance. These include intrapulmonary, mediastinal, retroperitoneal, pelvic or bone tumors [1]. Although mini- 
mally invasive, biopsies are still associated with a degree of risk and pain that add a psychological burden for the patient, in addition to the already heavy anxiety related to their underlying disease $[2,3]$.

The procedures may be performed under local anesthesia, with or without analgosedation, depending on the operator's experience [4]. Although the pain is usually considered to be mild, it is important for the physician to have a clear impression about how it is perceived by the patient and also about the associated anxiety. Thereby, any unnecessary suffering may be avoided and the patient can be confidently reassured prior to the procedure.

While most of the studies concerning the procedural pain are focused on the biopsy of breast tumors [5-7] and diffuse liver disease [3,4-8,9], there is limited evidence regarding the biopsy of tumors outside these organs $[2,10,11]$. Moreover, only one retrospective study is available concerning both CT and US guided biopsies [11], whereas no study has investigated procedure-related anxiety and discomfort depending on the guidance method employed. Therefore, the purpose of this study was to prospectively investigate the anxiety, pain, discomfort and other associated factors related to US- and CT-guided percutaneous biopsies in tumors outside the breast.

\section{Material and methods}

Approval of the institutional review board was obtained for this prospective study. All patients included in the study provided written informed consent for the interventional procedures and for the use of their data for research purposes according to the World Medical Association Declaration of Helsinki, revised in 2000, Edinburgh.

\section{Procedure details}

All the patients included in the study had a suspected malignant tumor that required histological confirmation. The indication for a biopsy was set by the referring physician or by an interdisciplinary tumor board. All the procedures were performed by the same radiologist with 4 years of experience in US- and CT-guided interventional procedures. The decision whether to use US or CT guidance was taken by the radiologist based on the site and US visibility of the tumor. In all cases, a CT or magnetic resonance imaging (MRI) examination was first performed in order to locate the safest biopsy route and, in the case of metastatic disease, the most accessible lesion. If a lesion was deemed approachable via US guidance, the patient underwent an US examination to confirm tumor visibility and access. If the lesion was not accessible by US, the patients were scheduled for a CT-guided biopsy. When possible, US guidance was preferred due to the lack of radiation and workflow considerations.
All biopsies were performed using a semiautomatic $18 \mathrm{G}$ core needle biopsy system with lengths of 10/15/20 $\mathrm{cm}$ and a corresponding $17 \mathrm{G}$ coaxial sheath with lengths of 5/10/15 cm (Somatex Medical Technologies, Berlin, Germany). US guidance was performed using a Logiq S7 machine (General Electric, USA) with either a broadspectrum convex transducer $(1.8-5 \mathrm{MHz})$ or a broadspectrum linear transducer (5-15 MHz). CT guidance was performed using a 64 slice GE Optima CT660 machine (General Electric, USA).

The coagulation status was verified before each procedure and had to be in compliance with the coagulation management guideline for percutaneous image-guided interventions [12]. If necessary, anticoagulant and antiplatelet therapy was ceased prior to the intervention as recommended by the guideline.

Before performing an US-guided biopsy, the region of interest was thoroughly scanned in order to find the safest approach. The patient was positioned accordingly and was instructed not to move throughout the procedure. The patient was covered in sterile drapes, the puncture site was disinfected and the transducer was inserted in a sterile glove. Local anesthesia of the skin and the planned puncture tract was performed using $10 \mathrm{ml}$ Lidocaine $1 \%$. The needle was inserted into the tumor using the freehand technique with longitudinal guidance.

Prior to performing a CT-guided biopsy, an unenhanced planning $\mathrm{CT}$ of the region of interest was performed. Contrast agent was administered only if the presence of larger vessels was suspected in the vicinity of the target lesion. Based on the planning CT, the patient position and the puncture path were chosen and marked and the patient was instructed to maintain the same position. After covering the patient in sterile drapes and performing local anesthesia, the needle was inserted into the tumor in a stepwise manner, using single-slice or tripleslice scans for guidance. For both CT and US-guided biopsies, between one and five samples were taken using the coaxial technique.

After the procedures, the patients were monitored on the ward and discharged on the following day in the absence of complications.

\section{Study design}

Sixty consecutive patients were included in the study between March 2018 and May 2019 and among which 24 (40\%) underwent CT-guided biopsies and $36(60 \%)$ USguided biopsies. The location of each biopsy is shown in table I.

Two to 6 hours after the procedure, a questionnaire was handed and explained to the patients by an investigator other than the performing physician. The patients were assured that their anonymous responses would be 
Table I. Biopsy sites

\begin{tabular}{lll}
\hline & US & CT \\
\hline Lung & 4 & 20 \\
Liver & 16 & 0 \\
Mediastinum & 0 & 1 \\
Kidney & 1 & 0 \\
Peritoneum & 3 & 0 \\
Retroperitoneum & 1 & 2 \\
Pelvis & 6 & 0 \\
Bone & 1 & 1 \\
Superficial tumors & 4 & 0 \\
\hline
\end{tabular}

US, ultrasonography; CT, computed tomography

used only for research purposes and they were encouraged to offer truthful answers.

The questionnaire (Supplementary Annex 1, on the journal site) consisted of six questions referring to their age, gender, whether it was the patient's fist percutaneous biopsy, whether any analgesic was taken before or after the procedure and whether the patient would have the procedure repeated if necessary. In addition, numerical rate scales (0-10) were used to quantify: biopsy-related anxiety, result-related anxiety, the quality of the information provided prior to the procedure, position-related discomfort, phlebotomy-related pain, procedural pain, post-procedural pain (until the time of filling in the questionnaire), overall satisfaction related to the procedure.

\section{Statistical analysis}

The results are presented as a mean \pm standard deviation. Statistical analysis was performed using GraphPad Prism 8. Numerical variables collected using the questionnaire were compared between the US and CT groups using the Mann Whitney test. Nominal variables were compared using the Fisher test. After pooling both US and CT groups together, Spearman analysis was used to determine variables that were correlated with procedural and post-procedural pain. The Kruskal-Wallis test for multiple comparisons was used to determine whether a greater degree of pain was reported for certain biopsy locations. The results were considered significant if $\mathrm{p}<0.05$.

\section{Results}

\section{Comparison between CT- and US-guided biopsies}

The age of the patients in the CT group was significantly higher than of those in the US group (63.5 \pm 10.6 years vs $54.7 \pm 13.9$ years, $\mathrm{p}=0.03$ ). In the $\mathrm{CT}$ group, the patients were preponderantly male $(65 \%, n=16)$, whereas in the US group the patients were preponderantly female $(75 \%, n=27)$. There was no significant difference $(p=0.2)$ regarding the presence of a previous biopsy between the CT group $(25 \%, n=6)$ and the US group (41.6\%, $n=15)$.
Immediately prior to the biopsy, $4.1 \%(n=1)$ of CT patients and $22.2 \%(n=8)$ of US patients received an analgesic drug $(p=0.07)$. After the biopsy, $16.6 \%(n=4)$ of CT patients and $5.5 \%(n=2)$ of US patients received an analgesic drug $(\mathrm{p}=0.2)$.

Regarding the variables collected using the numerical rate scales (Table II), there was no significant difference between the CT and US groups, except for the positionrelated discomfort. Patients rated the position during CT procedures (2.5 \pm 2.7$)$ significantly less comfortable $(\mathrm{p}=0.003)$ than during US procedures $(0.9 \pm 2.1)$. Among the CT procedures, $66.6 \%(\mathrm{n}=16)$ were performed in ventral decubitus, $29.1 \%(n=7)$ in dorsal decubitus and $4.1 \%(n=1)$ in lateral decubitus. Among the US procedures, $56.6 \%(\mathrm{n}=21)$ were performed in dorsal decubitus, $27.7 \%(n=10)$ in lateral decubitus, $8.3 \%(n=3)$ in ventral decubitus and $5.5 \%(n=2)$ in sitting position. All patients except one patient from every group reported that they would repeat the procedure if necessary.

\section{Procedural and post-procedural pain}

After pooling both groups together, the average procedural pain scores reported by the location of the lesion were: lung \& mediastinum 2.5 \pm 3.6 ; liver $1.3 \pm 1.4$; pelvis $2.0 \pm 1.5$; retroperitoneum $4 \pm 1$; peritoneum $0.3 \pm 0.5$; superficial tumors $3.5 \pm 1.7$; bone 0 . The post-procedural average scores were as follows: lung \& mediastinum $1.7 \pm 2.4$; liver $0.8 \pm 1.4$; pelvis $1.6 \pm 3.6$; retroperitoneum 0 ; peritoneum 0 ; superficial tumors $3.7 \pm 2.8$; bone 0 (fig 1). There were no significant differences between the body regions for both procedural and post-procedural pain. No procedural pain was reported by 18 patients $(30 \%)$.

Table II. Comparisons between computer tomography (CT) and ultrasonography (US) groups regarding the scores reported for each question included in the questionnaire

\begin{tabular}{lllll}
\hline Parameter & CT+US & $\mathbf{C T}$ & US & $\begin{array}{l}\text { p } \\
\text { (CT vs US) }\end{array}$ \\
\hline $\begin{array}{l}\text { Procedure } \\
\text { anxiety }\end{array}$ & $3.6 \pm 2.9$ & $3.1 \pm 2.5$ & $4.0 \pm 3.1$ & 0.3 \\
$\begin{array}{l}\text { Result } \\
\text { anxiety }\end{array}$ & $5.3 \pm 3.3$ & $4.6 \pm 3.4$ & $5.8 \pm 3.2$ & 0.1 \\
$\begin{array}{l}\text { Preprocedural } \\
\text { information }\end{array}$ & $9.7 \pm 1.1$ & $9.7 \pm 1.0$ & $9.6 \pm 1.2$ & 0.6 \\
$\begin{array}{l}\text { Position } \\
\text { discomfort }\end{array}$ & $1.5 \pm 2.5$ & $2.5 \pm 2.7$ & $0.9 \pm 2.1$ & 0.003 \\
$\begin{array}{l}\text { Phlebotomy } \\
\text { pain }\end{array}$ & $0.8 \pm 1.5$ & $0.7 \pm 1.2$ & $0.9 \pm 1.6$ & 0.9 \\
$\begin{array}{l}\text { Procedural } \\
\text { pain }\end{array}$ & $2.0 \pm 2.1$ & $2.0 \pm 2.2$ & $2.0 \pm 2.1$ & 0.9 \\
$\begin{array}{l}\text { Postprocedural } \\
\text { pain }\end{array}$ & $1.3 \pm 2.2$ & $1.5 \pm 2.4$ & $1.2 \pm 2.2$ & 0.3 \\
$\begin{array}{l}\text { Overall } \\
\text { satisfaction }\end{array}$ & $9.5 \pm 1.5$ & $9.2 \pm 2.2$ & $9.7 \pm 0.8$ & 0.5 \\
\hline & & & & \\
\hline
\end{tabular}


Only three patients reported pain scores higher than 5 , all of them having lung biopsies (fig 2). In the postprocedural interval, a higher proportion of patients reported no pain $(58.3 \%, \mathrm{n}=35)$ and four patients reported pain scores higher than 5 (fig 2). The procedural pain (2.0 \pm 2.1$)$ was significantly higher than the phlebotomy pain $(0.8 \pm 1.5, \mathrm{p}<0.0001)$ and the post-procedural pain $(1.3 \pm 2.2, \mathrm{p}=0.006)$.

Spearman analysis of the two groups pooled together showed that procedure-related anxiety $(\mathrm{p}=0.005)$, position-related discomfort (0.01), phlebotomy pain (0.0008) and the use of an analgesic before biopsy (0.02) were significantly correlated with the procedural pain. The other variables showed no significant correlation. The only factor that was significantly correlated $(p=0.007)$ with the post-procedural pain was the procedural pain (Table III).

\section{Discussions}

The only significant difference between CT and US-guided biopsies was noted for position-related discomfort, which was higher in the CT group and could be explained by the fact that two thirds of these patients had to lie in ventral decubitus. The duration of the procedures was not recorded, however, in our service CT-guided procedures usually lasted between 30 and 60 minutes, whereas US-guided procedures lasted between 15 and 30 minutes, including patient preparation. The longer duration of the CT-guided procedures could explain the discomfort, especially in the elderly. Anxiety, procedural and post-procedural pain and overall satisfaction evidenced no difference between US and CT groups. There

Table III. Correlation matrix for procedural and post-procedural pain

\begin{tabular}{lllll}
\hline & \multicolumn{2}{l}{$\begin{array}{l}\text { Procedural } \\
\text { pain }\end{array}$} & \multicolumn{2}{l}{$\begin{array}{l}\text { Post-procedural } \\
\text { pain }\end{array}$} \\
\cline { 2 - 5 } & $\mathbf{p}$ & $\mathbf{r}$ & $\mathbf{p}$ & $\mathbf{r}$ \\
\hline Age & 0.7 & -0.04 & 0.9 & 0.01 \\
Gender & 0.5 & 0.07 & 0.2 & -0.1 \\
Previous biopsies & 0.5 & 0,07 & 0.5 & 0.07 \\
Procedure anxiety & 0.005 & 0.3 & 0.1 & 0.2 \\
Result anxiety & 0.2 & 0.1 & 0.3 & 0.1 \\
Information quality & 0.5 & -0.07 & 0.3 & -0.1 \\
Position discomfort & 0.01 & 0.3 & 0.2 & 0.1 \\
Phlebotomy pain & 0.0008 & 0.4 & 0.1 & 0.2 \\
Procedural pain & 0 & 1 & 0.007 & 0.3 \\
Analgesia before biopsy & 0.02 & -0.2 & 0.4 & -0.1 \\
Analgesia after biopsy & 0.5 & 0.07 & 0.4 & 0.1 \\
\hline
\end{tabular}

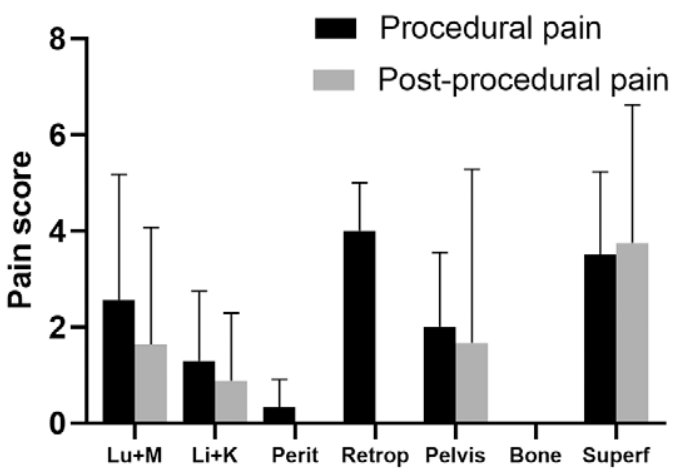

Fig 1. Pain scores reported by tumor location: comparison between procedural and post-procedural pain (Lu - Lung; M - Mediastinum; Li - Liver; K - Kidney; Perit - Peritoneum; Retrop - Retroperitoneum; Superf - Superficial tumors)

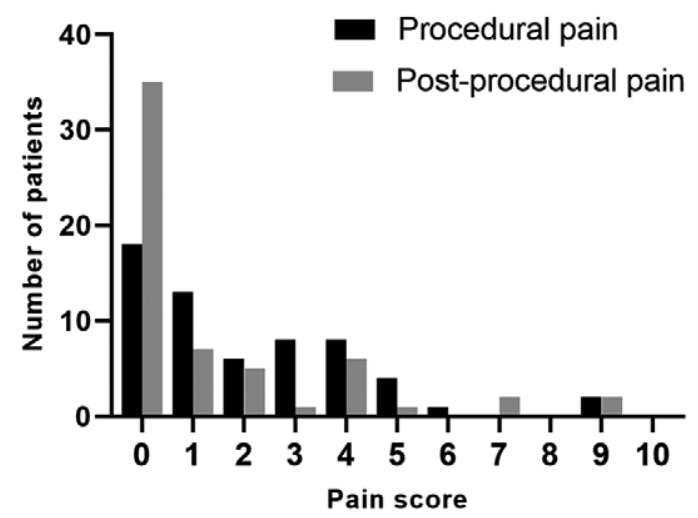

Fig 2. Patient distribution by reported pain score: comparison between procedural and post-procedural pain.

was a significant disparity between the CT and US groups in the distribution of age and gender, with the patients in the CT group being older and predominantly male. This is most likely explained by the fact that almost all of the CT-guided procedures were performed for the diagnosis of lung tumors and that lung cancer is more frequent in the male population, with a median age at diagnosis of 70 years [13]. In contrast, most biopsies in the US group were performed for hepatic metastases in patients with breast cancer, who are usually younger at first diagnosis [14].

Almost one third of the patients reported no procedural pain and more than half of the patients reported scores $\leq 2$. Only three patients reported pain levels higher than 5. The average procedural pain score was 2.0 in both CT and US groups. These scores were slightly higher than those caused by a phlebotomy which was rated on average with 0.8 . These findings are in line with other reports. In the case of liver biopsies the scores reported varied between 1.0 and $2.8[2-4,8,15]$. However, Iyer et al mentioned that $73.2 \%$ of patients reported no pain at 
all in a series of hepatic and extra-hepatic biopsies [11]. Mueller et al reported scores similar to ours (2.2) in patients with conscious sedation, but higher (4.3) under local anesthesia [2].

The pain decreased in the post-procedural interval to an average score of 1.3, with more than half of the patients reporting no pain. Eisenberg et al demonstrated an initial postprocedural increase in pain following hepatic biopsies, reaching the maximum at 30 minutes, which was followed by a decrease below the procedural level at 60 minutes. The decrease lasted for $240 \mathrm{~min}$, the pain level being steady afterwards at values less than 2 [8]. We recorded the post-procedural pain between 2 and 6 hours after the biopsy and recorded very similar values.

The procedural pain was not significantly different between body regions. It can be noted that the liver biopsies tended to be less painful with an average score of 1.3 and the retroperitoneal and superficial biopsies more painful with scores of 4 and 3.5, respectively. However, the results in these categories are unreliable due to the low number of patients. The pain associated with lung biopsies has been less investigated [10,11]. In our study, patients reported an average score of 2.5, not significantly different from that of liver biopsies, which is similar to the observations of Iyer et al [11]. Similarly, none of the patients questioned by Wong et al reported noteworthy biopsy-related pain, but considered that the most significant discomfort was related to the positioning in the CT scanner [10].

In a survey regarding the practices of liver biopsy in France, it was reported that $46 \%$ of the procedures were performed under analgosedation [4]. According to our experience, in the majority of cases, properly performed local anesthesia offers satisfactory pain relief for the patients. However, occasionally some patients may benefit from analgosedation. The factors that showed a positive correlation with the procedural pain were procedure-related anxiety, position-related discomfort and phlebotomy pain. The positive correlation with the pain experienced during a phlebotomy is an indicator of a reduced pain threshold which has been demonstrated to be influenced by anxiety in some patients [16]. Other authors have also shown that the pain expected by the patients was higher than the pain they actually experienced and that anxious patients tended to report higher pain scores $[3,8]$. The correlation between procedure-related pain and the position-related discomfort might also be a consequence of the decreased pain threshold. The post-procedural pain was significantly correlated only with the procedural pain.

The biopsies were generally accepted by the patients as a necessary step towards their diagnosis, and they felt more anxiety related to the result of the biopsy than to the biopsy itself. Despite the anxiety and pain, the overall satisfaction of the patients regarding the procedure was high, the majority being willing to repeat the procedure if necessary. Only two patients experienced severe pain, both reporting scores of 9 and their unwillingness to repeat the procedure if necessary. We consider that these patients might have benefited from the administration of analgosedation.

A limitation of this study is the relatively low number of patients with biopsies in locations other than the liver and lung, resulting in a heterogeneous population and in the lack of possibility to analyze these subgroups individually. Another source of bias may be the time point at which the patients were questioned. The fact that the anxiety level was investigated after the biopsy had taken place might have influenced the responses based on the painfulness of the procedure. The procedure-related pain was also investigated a few hours after the biopsy was performed, which could have been influenced by the post-procedural pain. However, we consider that these sources of error are minimal as our results are similar with previous reports.

\section{Conclusions}

Our study has shown that most of the percutaneous biopsies were associated with low and moderate levels of pain, with only individual cases of severe pain. The pain was not influenced by the guidance method, nor by the body region that was biopsied, but was significantly correlated with the anxiety level, the pain experienced during a phlebotomy and the position-related discomfort. The patients showed high overall satisfaction and were willing to repeat the procedure if needed.

\section{Conflict of interest: none}

\section{References}

1. Roman A, Achimas-Cadariu P, Fetica B, Gata V, Seicean A. CT-guided procedures: an initial experience. Clujul Med 2018;91:427-434.

2. Mueller PR, Biswal S, Halpern EF, Kaufman JA, Lee MJ. Interventional radiologic procedures: patient anxiety, perception of pain, understanding of procedure, and satisfaction with medication--a prospective study. Radiology 2000;215:684-688.

3. Akay S, Karasu Z, Noyan A, et al. Liver biopsy: is the pain for real or is it only the fear of it? Dig Dis Sci 2007;52:579-581.

4. Cadranel JF, Rufat P, Degos F. Practices of liver biopsy in France: results of a prospective nationwide survey. For the Group of Epidemiology of the French Association for the Study of the Liver (AFEF). Hepatology 2000;32:477-481. 
5. Zografos GC, Zagouri F, Sergentanis TN, et al. Pain during vacuum-assisted breast biopsy: are there any predictors? Breast 2008; 17:592-595.

6. Zagouri F, Sergentanis TN, Gounaris A, et al. Pain in different methods of breast biopsy: emphasis on vacuum-assisted breast biopsy. Breast 2008;17:71-75.

7. Pang E, Crystal P, Kulkarni S, Murphy K, Menezes RJ. An Audit of Pain Experienced During Image-Guided Breast Biopsy Procedures at an Academic Center. Can Assoc Radiol J 2016;67:250-253.

8. Eisenberg E, Konopniki M, Veitsman E, Kramskay R, Gaitini D, Baruch Y. Prevalence and Characteristics of Pain Induced by Percutaneous Liver Biopsy. Anesth Analg 2003;96:1392-1396.

9. Mahadeva S, Mahfudz AS, Vijayananthan A. Ethnicity influences pain after ultrasound-guided percutaneous liver biopsy. Eur J Gastroenterol Hepatol 2015;27:1378-1381.

10. Wong ET, Dunham C, Patsios D. Qualitative assessment of pain management in patients undergoing computed tomography-guided transthoracic lung biopsy. Pain Res Manag 2014;19:149-152.
11. Iyer VR, Sheedy SP, Gunderson TM, et al. Procedure-Related Pain During Image-Guided Percutaneous Biopsies: A Retrospective Study of Prevalence and Predictive Factors. AJR Am J Roentgenol 2019;213:755-761.

12. Patel IJ, Davidson JC, Nikolic B, et al. Consensus guidelines for periprocedural management of coagulation status and hemostasis risk in percutaneous image-guided interventions. J Vasc Interv Radiol 2012;23:727-736.

13. de Groot PM, Wu CC, Carter BW, Munden RF. The epidemiology of lung cancer. Transl Lung Cancer Res 2018;7:220-233.

14. Key TJ, Verkasalo PK, Banks E. Epidemiology of breast cancer. Lancet Oncol 2001;2:133-140.

15. Tan KT, Rajan DK, Kachura JR, Hayeems E, Simons ME, Ho CS. Pain after percutaneous liver biopsy for diffuse hepatic disease: a randomized trial comparing subcostal and intercostal approaches. J Vasc Interv Radiol 2005;16:12151219.

16. Robin O, Vinard H, Vernet-Maury E, Saumet J. Influence of sex and anxiety on pain threshold and tolerance. Funct Neurol 1987;2:173-179. 